\title{
Estudio Descriptivo Sobre La Influencia De Las Variables Personales Y El Fracaso Escolar En La Ciudad Autónoma De Melilla
}

\author{
Dña. $M^{a}$ del Mar Estrada de Madariaga \\ Licenciada en Psicopedagogía, Maestra en Lengua extranjera (Inglés) \\ Orientadora educativa del MECD, Melilla (España) \\ Dr. D. Juan Manuel Trujillo Torres \\ Diplomado en Magisterio de Educación Física, Licenciado en Pedagogía, \\ Doctor en Ciencias de la Educación y Máster universitario en Nuevas \\ Tecnologías aplicadas a la educación \\ Profesor Titular en el Dpto. de Didáctica y \\ Organización Escolar de la Universidad de Granada. \\ Universidad de Granada. Facultad de Ciencias de la Educación, \\ Granada (España)
}

doi: 10.19044/esj.2016.v12n16p37 URL:http://dx.doi.org/10.19044/esj.2016.v12n16p37

\begin{abstract}
The analysis of this study has been approached through a questionnaire. The questionnaire consists of two parts. The first is personal and academic data. The second part of the questionnaire contains 46 items. It is a Likert scale of four alternatives. For the statistical treatment of the data it was used SPSS v.21 for Windows. For the two-dimensional descriptive statistical analysis it was used the Mann-Whitney and Kruskal-Wallis programs. The most relevant contributions, in which the theoretical framework of this research is based, have been the Diagnostic and Statistical Manual of Mental Disorders, 2014 (DSM-5); Educational Law, especially that related to diversity, as well as the different contributions of studies and doctoral theses related to the investigation. From this study, intervention proposals and new lines of research will be carried out in order to reduce the high rate of failure and early school dropout in this city.
\end{abstract}

Keywords: Learning motivation, self esteem, learning difficulties, anxiety

\section{Resumen}

El análisis de este estudio se ha abordado a través de un cuestionario. El cuestionario consta de dos partes diferenciadas. La primera corresponde a los datos personales y académicos. La segunda parte del cuestionario consta 
46 ítems . Es una escala Likert, de cuatro alternativas. Para el tratamiento estadístico de los datos se ha utilizado el SPSS v.21 para Windows. Para el análisis estadístico descriptivo bidimensional se utilizaron la prueba de Mann-Whitney y Kruskal-Wallis. Las aportaciones más relevantes y que fundamentan el marco teórico de esta investigación han sido el Manual Diagnóstico y Estadístico de los Trastornos Mentales de 2014 (DSM-5); la legislación actual, en especial la relacionada con el tratamiento a la diversidad; así como las diferentes aportaciones de estudios y tesis relacionados con el tema de investigación. A partir de este estudio se realizarán propuestas de intervención y se plantearán nuevas líneas de investigación con el fin de disminuir la elevada tasa de fracaso y abandono escolar temprano en esta ciudad.

Palabras Clave: Motivación para el aprendizaje, autoestima, dificultades de aprendizaje, ansiedad

\section{Introducción}

El objetivo del presente estudio es analizar las variables personales que contribuyen de forma más significativa al fracaso y abandono escolar temprano en la Ciudad Autónoma de Melilla, realizándose un diagnóstico previsible de los factores personales que afectan al fracaso escolar.

La población del Melilla está constituida por 83.700 personas. De acuerdo al informe de Datos y Cifras del curso 2015-2016 (Subdirección de Estadística y Estudios del Ministerio de Educación, Cultura y Deporte, 2015) en la Ciudad Autónoma tenemos un alto índice de abandono escolar temprano, situado en un $24,7 \%$. Por lo que se refiere al rendimiento en matemáticas, lectura y ciencias, el informe PISA 2012, nos sitúa en España con malos resultados respecto a los países de la OCDE y a Melilla con resultados de los más bajos respecto al resto de Comunidades españolas.

En cuanto al contexto educativo y familiar, hay que destacar que una gran parte del alumnado matriculado en los centros escolares es de origen bereber cuya lengua materna es el tamazight, lo que dificulta el desarrollo de la competencia lingüística en castellano de los alumnos y alumnas. Por lo que se refiere al contexto familiar, es de resaltar que hay un alto índice de familias que provienen de entornos socio-culturales y económicos desfavorecidos, donde el índice de analfabetismo es grande, sobre todo entre las mujeres. 


\section{Texto principal}

\section{Fundamentación teórica}

Se ha tomado como referente normativo la Ley Orgánica 8/2013, para la mejora de la calidad educativa (LOMCE) y la Orden EDU 849 de 2010 relativa a la atención a la diversidad.

Nuestro marco teórico en relación a las variables personales que afectan al rendimiento académico se fundamenta en los estudios más relevantes de la historia de la psicología; así como en investigaciones actuales sobre los factores afectivo-emocionales que inciden en el rendimiento escolar (Ferguson, 2000; Petri, H. L. Govern, J. M. 2006; Pintrich, 2003; Rodríguez Fuentes, 2009; Rosenthal y Jacobson, 1968; Ortega Ruiz, 2001; O’Moore y Kirkham, 2001 y Fernández Castillo \& Gutiérrez Rojos, 2009. Hemos utilizado la Guía de consulta de los criterios diagnósticos del DSM-5, de 2014 en referencia al estudio de determinados trastornos. La aportaciones del Informe PISA 2012 nos servirán para contrastar o corroborar datos obtenidos en este estudio.

\section{Método}

La población del estudio está compuesta por 813 estudiantes del Centro de Enseñanza de Personas Adultas Carmen Conde, tanto de enseñanzas iniciales como de la ESO y por 639 usuarios de la Unidad de Orientación del Convenio MECD con la Ciudad Autónoma de Melilla. La muestra de los participantes en la investigación está constituida por 93sujetos, 35 de la Unidad de Orientación y 64 del CEPA Carmen Conde. Cabe destacar que el porcentaje de mujeres participantes en el estudio es ligeramente superior al de hombres, con un $59,1 \%$ y $40,9 \%$ respectivamente. El intervalo de edad más representativo de la muestra se sitúa entre los 16 y 21 años, con un porcentaje del 31,2\%; seguido del intervalo de edades comprendidas entre 22 y 27 años, cuyo porcentaje se halla en el 20,4\%. Respecto al nivel máximo de estudios alcanzados cabe mencionar que la muestra está compuesta en su gran mayoría por personas con estudios primarios, un 20,4\% y aquellos que abandonaron el sistema educativo en cursos de $2^{\circ}$ y $3^{\circ}$ de Educación secundaria obligatoria, con un 21,5\% cada uno. 


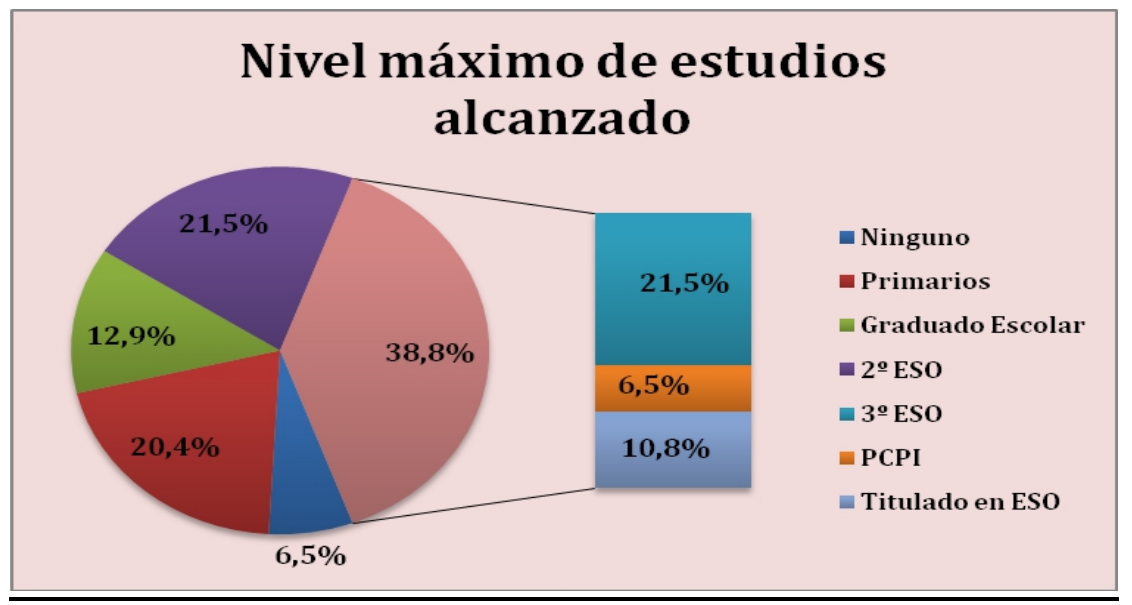

Figura $\mathrm{n}^{\circ} 1$. Nivel de estudios alcanzado por la muestra del estudio.

El porcentaje de personas que presenta algún tipo de discapacidad que no sea intelectual, física o sensorial, apenas representativa, es de tan solo el $8,5 \%$. Solo el $17,2 \%$ de los encuestados indican que padecían una enfermedad y que ésta fue la causa por la que no iniciaron o continuaron estudios. Respecto a los estudios alcanzados por el padre cabe destacar que hay un porcentaje muy signifcativo que carece de estudios, el $57 \%$; seguidos de un $20 \%$ que solo han cursado estudios primarios. Respecto a los estudios máximos alcanzados por la madre, los resultados del análisis de las encuestas indican que en su gran mayoría carece de estudios, un 58,1\%, porcentaje ligeramente superior al de los padres.

\section{Hipótesis:}

\section{Instrumento}

Para el diseño del cuestionario se han tenido presentes los objetivos que se persiguen en la investigación.

Para su elaboración se partió de las variables necesarias para dar respuesta a los objetivos planteados relacionados con la motivación, las atribuciones, las expectativas, las dificultades de aprendizaje y la ansiedad.

El cuestionario consta de dos partes diferenciadas. La primera corresponde a los datos personales y académicos, en los que consta género, edad, estudios alcanzados, centro donde curso estudios, discapacidad, enfermedad; así como estudios de la madre y del padre. La segunda parte del cuestionario consta de aquellos ítems relacionados con las variables internas que afectan al rendimiento escolar. Es un cuestionario compuesto por 46 ítems cerrados, de tipo Likert, de cuatro alternativas.

La pasación del cuestionario se realizó en febrero y marzo de 2015. Antes de pasar el cuestionario a los participantes en el estudio, se les informó 
de los objetivos del estudio; así como de que su realización era voluntaria. En algunos casos, se pasó el cuestionario de forma individual, aclarando las dudas y facilitando la comprensión de los diferentes ítems. En los casos en que ha sido posible se ha pasado de forma colectiva.

\section{Análisis de datos}

Las preguntas relativas a los datos personales y académicos son de respuesta dicotómica y de razón. Las preguntas relacionadas con cada una de las variables objeto de estudio son ordinales de cuatro niveles (totalmente de acuerdo, de acuerdo, en desacuerdo, totalmente en desacuerdo).

Para el tratamiento estadístico de los datos se ha utilizado el paquete estadístico SPSS v.21 para Windows. Para el análisis estadístico descriptivo bidimensional se utilizaron una serie de estadísticos como la prueba de Mann-Whitney para variables dicotómicas y Kruskal-Wallis para aquellas de naturaleza ordinal y policotómica. En concreto, se pretenden buscar relaciones de dependencia con las variables referidas al género, edad, nivel máximo de estudios alcanzado, discapacidad y/o enfermedad, centro donde cursaron los estudios, nivel de estudios de los padres y los factores intrapersonales.

La prueba de U Mann-Whitney, se utilizó para la comprobación de dos muestras donde la variable independiente es dicotómica y la escala de medida de la variable dependiente es de naturaleza ordinal. Las observaciones de ambos grupos se combinaron y adecuaron con el rango promedio en el caso de pares (Mann y Whitney, 1947). En cuanto a la prueba no paramétrica $\mathrm{H}$ de Kruskal-Wallis, siendo una extensión de la prueba de U Mann-Whitney, es el equivalente a la prueba paramétrica del análisis de varianza de una vía. Nos permite conocer si hay diferencias en las distribuciones de la variable. Las premisas que asumimos para su uso son que la variable dependiente es ordinal con más de dos niveles y la dependiente de carácter cuantitativo, no hay mediciones repetidas ni categorías de respuestas múltiples (Kruskal, 1941; Kruskal, 1952; Kruskal, 1958).

\section{Fiabilidad y validez del cuestionario.}

Respecto a la validez y fiabilidad del instrumento se ha seguido el juicio de expertos (validez de contenido). También una validez semántica contrastando diversas perspectivas de significación. Todo ello realizado por profesionales de diferentes Universidades españolas.

La fiabilidad del instrumento queda reflejada a través de dos pruebas, la de alfa de Cronbach obteniendo el valor numérico $\alpha=0.919$ y la prueba de las dos mitades de Guttman cuyo resultado ha sido de 0.865. Ambos 
valores, se consideran muy buenos, pues indican una fiabilidad alta del cuestionario construido.

\section{Resultados}

A continuación, se irán exponiendo los resultados en función de los objetivos planteados y en orden decreciente:

Relacionado con la ansiedad ante situaciones de pruebas orales, exámenes, los resultados muestran que hay un 46,2\% de participantes que sufrían ansiedad ante los exámenes.

Es de destacar que hay un alto porcentaje de la muestra que presentaba dificultades específicas de aprendizaje, especialmente en el área de matemáticas. Concretamente, al $40,9 \%$ de los participantes le costaba realizar las cuentas matemáticas; al 39,8\% de los encuestados le resultaba difícil realizar los problemas y el $26,9 \%$ tenían dificultades de memoria relacionadas con la materia.

Por lo que se refiere a la lectura el 20,4\% de la muestra asume que tardó bastante en aprender a leer y el 22,6\% de los participantes opina que le costaba entender lo que leía.

En relación a la escritura el 32,3\% de la muestra asume que su escritura estaba llena de faltas de ortografía y el 19,4\% asume que era muy difícil entender su escritura.

Por otro parte es de destacar las dificultades relacionadas con la memoria; pues el 33,3\% de la muestra afirma que tardaba mucho en aprenderse los temas y el 26,9\% opina que le suponía mucho esfuerzo memorizar las tablas de multiplicar o el proceso necesario para la realización determinados cálculos matemáticos.

Por lo que se refiere a la motivación, hay que destacar que un 22,6\% de la muestra no atendía a las explicaciones del profesorado; además el $30,1 \%$ de los encuestados No preguntaba las dudas a los profesores y el 19,3\% de la muestra No llevaban a clase el material necesario. Es muy significativo que el 36,5\% de los encuestados afirma que no les gustaba estudiar. Por otro lado, el $36,6 \%$ de los encuestados afirman que estudiaban de memoria solo para aprobar el examen; y el $20.4 \%$ solo lo hacía para evitar castigos o que los padres se enfadaran.

Respecto a las atribuciones que hacen de éxitos y fracasos es destacable que el $28 \%$ de la muestra afirma que si le suspendían era porque no era capaz de entender las explicaciones, eran muy difíciles para é/ella. Igualmente, el 21,5\% de los encuestados cree que suspendía porque los exámenes eran demasiado difíciles para él/ella. En relación a las atribuciones externas de éxitos y fracasos destacan los siguientes resultados: el $12,9 \%$ de los participantes opina que aprobaba solo porque tenía suerte; el 11,9 de la muestra afirma que si aprobaba era porque el profesor/a era 
muy bueno con él/ella; el 12,9\% de los encuestados piensa que si suspendía era porque el profesor/a explicaba mal; el $8,6 \%$ de los participantes afirma que le suspendían porque tenía mala suerte y el $6,5 \%$ de la muestra opina que suspendía porque los profesores/as le tenían manía.

En relación con las expectativas, es de subrayar que el 24,7\% de los encuestados piensa que aunque se esforzara no iba a aprobar y el 17,3\% de la muestra está de acuerdo de que en el futuro los estudios no le servirían para nada.

Por los que se refiere a la autoestima y autoconcepto académico, hay que destacar que el $22,6 \%$ de los participantes opina que no valen para estudiar; el $21,6 \%$ de los encuestados no se considera inteligente. Asimismo, el 23,6\% de la muestra no se considera trabajador/a y el 11,5\% de los encuestados piensa que no le valoran, ni le aprecian.

En relación a la lengua materna, destaca el porcentaje de desconocimiento parcial o total de la lengua vehicular que es del 7,6\% y 7,5\% respectivamente.

En cuanto a los hábitos de estudio el 55,9\% de la muestra opina que no estudiaba todos los días, y el $46,3 \%$ asevera que no hacían las tareas a diario.

Respecto al absentismo el $10,4 \%$ de los participantes comenta que faltaban a clase porque se dejaban llevar por otros compañeros; el 10,8\% de los encuestados afirma que le expulsaban por mal comportamiento y esa era la razón por la que faltaba a clase. Igualmente, el 10,8\% de los participantes afirma que faltaban a clase porque tenían que ayudar en casa.

\section{Conclusión}

En relación a las variables internas al sujeto hay que destacar que uno de los factores que afectan de forma más significativa al rendimiento escolar es la ansiedad ante situaciones de pruebas orales, exámenes, los resultados muestran que hay un 46,2\% deencuestados que sufrían ansiedad ante los exámenes. La sintomatología asociada a la ansiedad y citando el DSM-5 del 2014 son nervisosismo, fatiga y dificultades para concentrarse. Por otro lado, es necesario incidir en que la ansiedad afecta a los niveles de atención, a consecuencia de lo que se producen dificultades de procesamiento de la información y esto afecta al rendimiento académico (Fernández Castillo, 2009). Este alto nivel de ansiedad puede estar provocado por la falta de hábitos de estudio; ya que el alumnado quiere aprobar los exámenes pero deja el estudio y preparación de los mismos para el último día, lo que conduce a que se bloqueen y no puedan concentrarse. Acerca de los hábitos de estudio hay que destacar que más del $55 \%$ de los participantes no estudiaba todos los días y cerca del $47 \%$ no hacía las tareas a diario. Concretamente una de nuestras hipótesis expresaba que la ansiedad influye 
de manera significativa en el alumnado que no tiene hábitos, ni buenas estrategias de estudio y que muestra interés por los estudios.

\section{Me ponía muy nervioso en los exámenes y me bloqueaba, no recordaba nada, $o$ muy poco de lo estudiado}

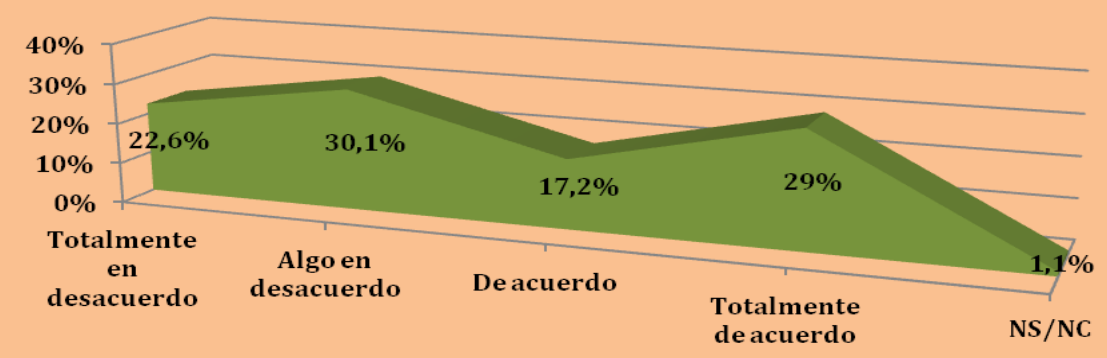

Figura $\mathrm{n}^{0} 2$. Relación ansiedad y rendimiento escolar

Otros factores con gran incidencia en el fracaso escolar son las dificultades específicas de aprendizaje, y entre éstas, como se ha podido observar en los resultados obtenidos en el análisis estadístico, las que influyen de manera más significativa son las dificultades relacionadas con las matemáticas.

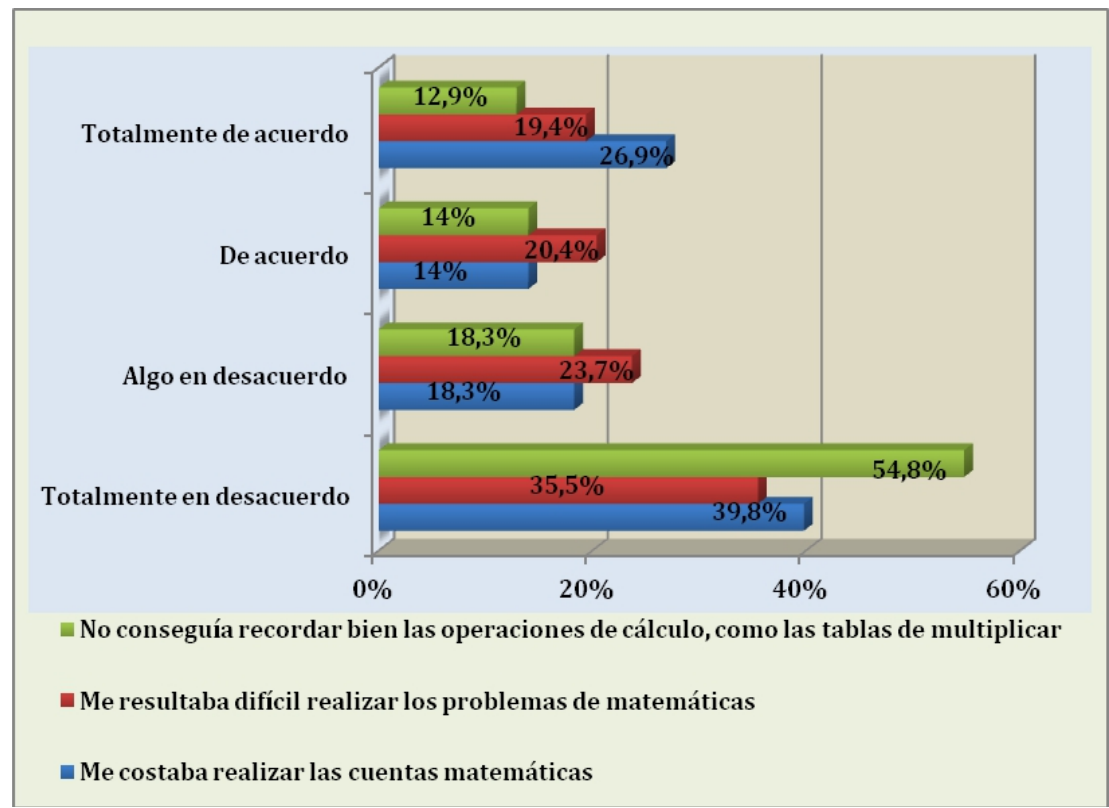

Figura $\mathrm{n}^{0} 3$. Dificultades de aprendizaje de las Matemáticas 
En cuanto al ítem del cuestionario relacionado con la facilidad que perciben respecto al cálculo en matemáticas, se ha encontrado una relación de dependencia respecto al nivel de estudios alcanzado. El 6,5\% de los participantes con estudios primarios opina estar en total acuerdo respecto a las dificultades de cálculo y el $8,6 \%$ de los que su nivel de estudios es $2^{\mathrm{a}}$ ESO.

En relación a la resolución de problemas matemáticos, nos encontramos que los estudiantes españoles utilizan más estrategias de memorización situándose por encima de la media de los países de la OCDE. Incidiendo en la relación de estrategias de memorización y la materia de matemáticas, hay que destacar que el informe PISA 2012 establece que este tipo de estrategias son eficaces para problemas sencillos, pero no para los complejos.

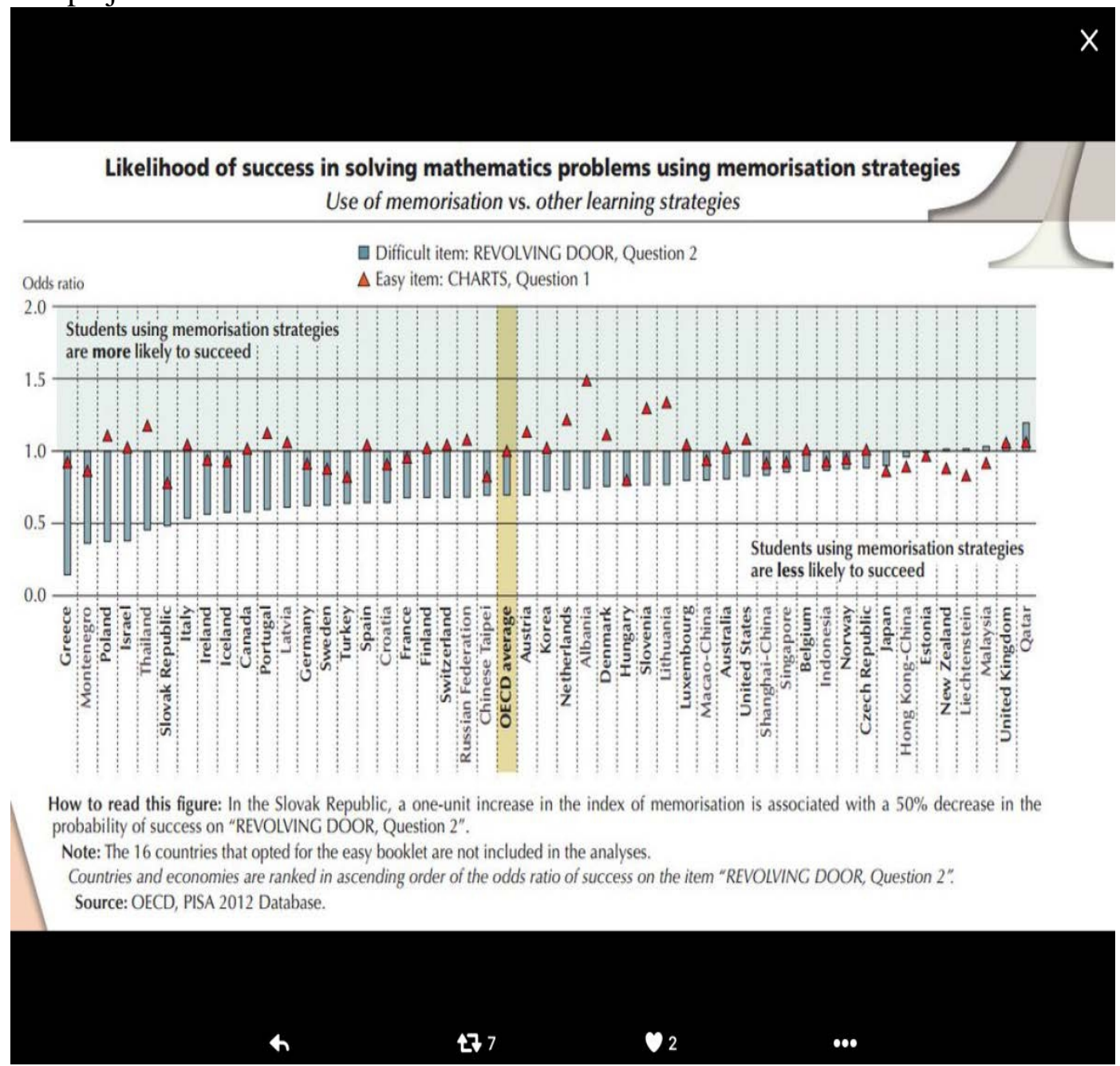

Figura $\mathrm{n}^{\circ}$ 4. Estrategias de memorización y los problemas matemáticos. 
Referente a la dificultad para realizar los problemas de matemáticas existe una relación de dependencia respecto al nivel de estudios alcanzados por los participantes del estudio. Son aquellos con un nivel de $2^{\circ}$ ESO donde hay un 4,3\% en el grado de acuerdo. Igualmente entre los participantes con estudios primarios hay un porcentaje igual en el grado de total acuerdo. En cuanto a estudios realizados en Ceuta y Melilla con jóvenes de 16 a 24 años, con o sin titulación de la ESO pero que no continuaron estudios, los resultados relacionados con el aprendizaje de las matemáticas muestran que el porcentaje de suspensos de esta materia es del 85\% en Melilla (Sánchez et al., 2008). En la misma línea se sitúan los estudios realizados por el INEE (MECD, 2013) que muestran que conseguimos resultados muy bajos en la competencia matemática en comparación con los países de la Unión Europea y de la OCDE. Los resultados PISA 2012 en contraposición a los obtenidos en 2003 en el área de matemáticas, destacan que España obtiene una baja puntuación, pero ha mejorado sus resultados. Es de destacar que, en relación al género, los chicos siempre han obtenido mejores resultados que las chicas en esta materia. Como resultado de las repeticiones de curso, hay que subrayar que los resultados y porcentajes de España en matemáticas entre los años 2003 al 2012 mejoran significativamente cuando el alumnado ha permanecido durante dos cursos más en la misma etapa. El informe PISA 2012 analiza asimismo, el rendimiento en matemáticas con la condición de ser inmigrante. El rendimiento en matemáticas de un inmigrante es inferior al rendimiento que puede tener el estudiante del país de origen. España obtiene un rendimiento por debajo de la Unión Europea y de los países de la OCDE; tanto si son alumnado inmigrante, como si son del país de origen.

En relación a las dificultades en la lectura, los resultados del estudio en el que se muestra claramente que hay dificultades en esta materia, reafirman lo demostrado en el informe PISA 2012 en los que España obtuvo unos bajos resultados. En relación a la repetición de curso, los resultados y porcentajes de España en lectura en los años 2000, 2009 y 2012, indican que se ve un incremento de la competencia lectora cuando hay dos repeticiones.

A continuación se comentarán los resultados obtenidos por tres de las hipótesis de partida relacionadas con la motivación, las atribuciones y las expectativas y su interrelación.

Varios son los autores que tratan la motivación y su implicación en el rendimiento de los sujetos. En este artículo queremos destacar las contribuciones de Pintrich (2003) para el que la motivación está relacionada con la dirección y activación del comportamiento y está vinculada con lo que el alumno percibe que es capaz de realizar de forma satisfactoria. Del mismo modo, Rodríguez Fuentes (2009) destaca que dependiendo de la valoración que dé el estudiante a las tareas, mayor o menor será su implicación en las mismas. Los resultados de este estudio destacan que al 36,5\% de la muestra 
no les gustaba estudiar. Existe una relación de dependencia en relación los participantes que han cursado estudios en un IES de la ciudad ubicado en el extrarradio. Estos se muestran en un grado de acuerdo del 6,5\%. Relacionado con los estudios de la madre nos encontramos que el 20,4\% de la muestra, cuyas madres carecen de estudios o solo tiene un certificado de haber estudiado, se muestran en total acuerdo o en acuerdo. Muchos de los estudiantes de bajo rendimiento opinan que estudiar es una pérdida de tiempo (Ortega Torres, 2006) y esto se ve corroborado por los resultados del estudio en los que un alto porcentaje de los encuestados asume que no preguntaban las dudas al profesorado y solo estudiaban para evitar castigos de los padres.

En cuanto a las atribuciones, el alumnado con malos resultados escolares suele hacer atribuciones de sus éxitos y fracasos que afectan a su rendimiento escolar. Como se ha podido constatar en los resultados del análisis estadístico, un alto porcentaje atribuye sus fracasos a la falta de capacidad, causa incontrolable e interna, y otros lo hacen a causas externas e incontrolables, lo que incide negativamente en la motivación (Weiner, 1986). Conviene subrayar que este alumnado suele atribuir los fracasos a poca capacidad, falta de esfuerzo, dificultad de la tarea o la mala suerte. García López (2006).

En relación a las expectativas, se observa un alto porcentaje que muestra expectativas negativas en relación a los estudios y a esto hay que añadir que perciben que el profesorado tiene expectativas bajas sobre su capacidad y rendimiento. Hay que incidir en la importancia de las expectativas mostradas por el profesorado y la relación con el éxito de sus alumnos, ya que cuando el profesorado tiene bajas expectativas respecto el rendimiento de su alumnado, estas acaban cumpliéndose; es el conocido "Efecto Pygmalión" (Rosenthal, 1980).

Por lo que se refiere a los hábitos de estudio, los resultados del análisis estadístico demuestran que el $40 \%$ de la muestra afirma que estudiaba a diario. Del análisis diferncial se constata que son las mujeres las que sobresalen por su constancia en el trabajo diario con un 32,3\%, frente al $10,8 \%$ de los hombres. 


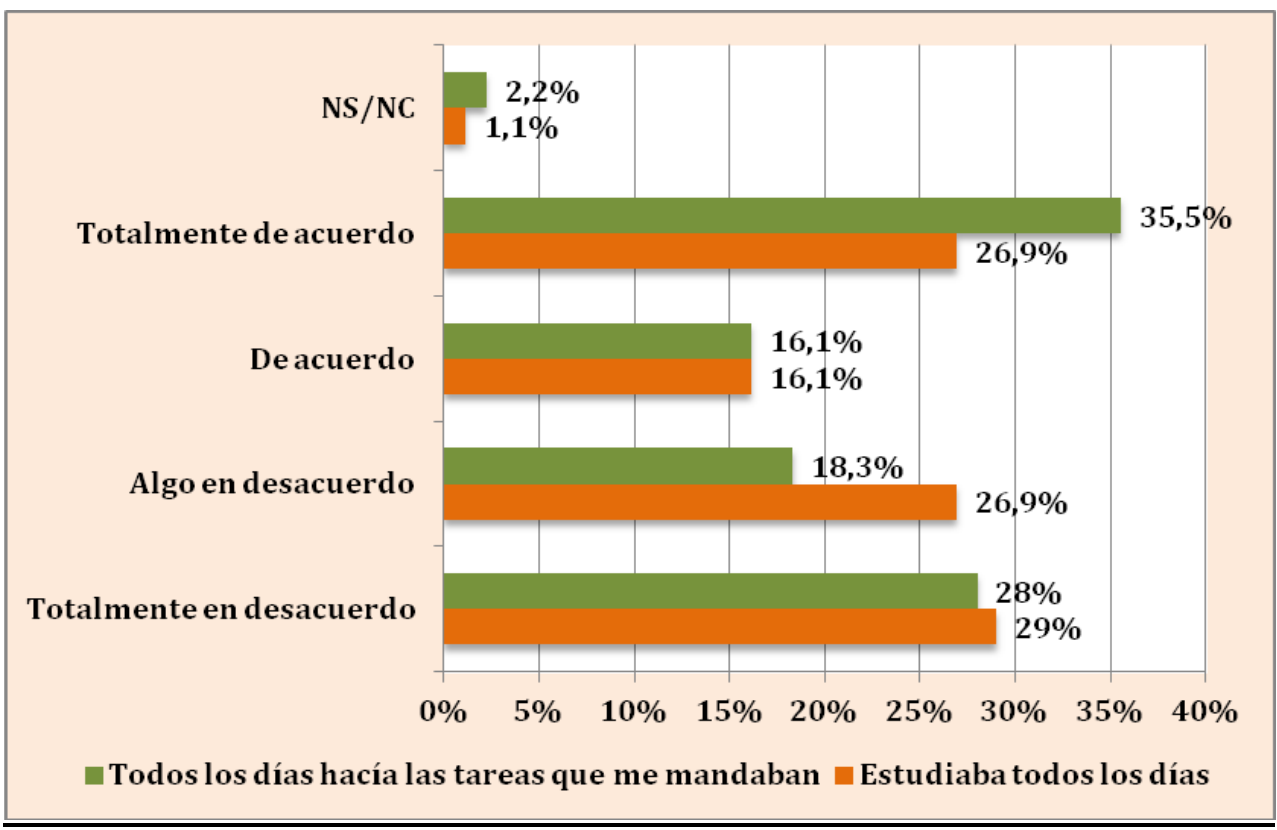

Figura $\mathrm{n}^{\circ}$ 5. Hábitos de estudio

La falta de hábitos de estudio queda reflejada asimismo en un estudio realizado por Sánchez et al., en el que se afirma que el $80 \%$ de los encuestados nunca acudían a la biblioteca para realizar trabajos o estudiar; el $69,9 \%$ no asistían a academias para recibir apoyo escolar y el 43,8\% casi nunca realizaba las tareas escolares fuera del horario escolar.

Conviene subrayar que respecto a la autoestima y su relación con el rendimiento escolar, que era la cuarta hipótesis planteada, en la adolescencia es un factor esencial para afrontar los estudios, ya que es un constructo que abarca el concepto que se tiene sobre la propia valía. Para Ortega Ruiz (2001) la autoestima representa el valor relativo que se atribuyen los sujetos o lo que perciben que los demás piensan sobre ellos. En particular, el 24\% de la muestra presenta un autoconcepto académico y una autoestima baja, como se ha podido comprobar en los resultados aportados en el apartado anterior. Asimismo, hay que subrayar que los adolescentes con baja autoestima tienen más conductas amenazantes e intimidatorias hacia otros (O’Moore y Kirkham, 2001), lo que afecta a la integración del alumnado en el aula; así como al clima de convivencia del aula, que son fundamentales a la hora de abordar los aprendizajes. En la tesis "La familia, la autoestima y el fracaso escolar del adolescente" se extraen conclusiones dignas de mención, como que la baja autoestima provoca situaciones de inseguridad, problemas de conducta relacionadas con el respeto, agresividad y que el adolescente empiece a presentar conductas no saludables (Carrillo Picazo, 2009). Este tipo de conductas incide negativamente en el rendimiento escolar; ya que 
como se ha expuesto éste se ve afectado por los hábitos de estudio, la ansiedad, la motivación y las expectativas, que decaen al presentar el adolescente este tipo de comportamientos.

Respecto a las dificultades de memoria, un alto porcentaje afirma que presenta dificultades a la hora de memorizar y recordar los contenidos de aprendizaje. Habría que relacionar estas dificultades con la falta de motivación y en especial con la falta de hábitos y estrategias de estudio ya que casi un $37 \%$ de la muestra alega que estudiaban de memoria solo para aprobar el examen.

En cuanto a las dificultades atencionales solo el 9,7\% de la muestra afirma que presentó problemas de atención. La experiencia como docentes nos dice que sesto se contradice con la realidad del aula, ya que es una de las mayores quejas del profesorado. Pueden que el ítem que realiza la pregunta sobre el nivel de atención lo enfoque a trastorno de atención diagnosticado. Cabe subrayar que los problemas atencionales suelen derivar de la falta de interés y motivación hacia la tarea.

En lo concerniente a la lengua materna en relación a la vehicular, son destacables los porcentajes obtenidos en el análisis estadístico en los que se concluye que el porcentaje de desconocimiento parcial o total de la lengua vehicular es del 7,6\% y 7,5\% respectivamente. Como se ha comentado en el análisis del contexto educativo, hay un gran porcentaje de alumnado de origen bereber cuya lengua materna es el tamazight. Si bien la superación de estas dificultades se produciría con la escolarización temprana, también hay que destacar que con la llegada de la crisis tenemos población que había emigrado a Cataluña o a países europeos y que ha regresado a Melilla en busca de trabajo. Sus hijos no hablan el castellano y o presentan muy baja competencia lingüística en este idioma. A esto hay que añadir que según el Sistema Estatal de Indicadores de la Educación (MECD, 2015), el mayor porcentaje de alumnado extranjero se encuentra matriculado en Ed. Secundaria Obligatoria, un 11,3\%. A estos datos hay que añadir que los extranjeros no pertenecientes a la Unión Europea censados en nuestra ciudad son 5.400 personas, de las cuales 1.900 son hombres, lo que supone un $35,1 \%$ y 3.500 mujeres, un 64,8\% (INE, 2016). Se puede estimar que en su gran mayoría son analfabetos, al menos para nuestro sistema educativo. Muchas de las mujeres proceden de pueblos de Marruecos. Cuando se les pasó el cuestionario en la Unidad de Orientación, algunas de ellas comentaron que carecían de estudios porque en "su pueblo no había escuela”; para otras, la razón era que en Marruecos “las niñas no estudian, se quedan trabajando en las tareas de casa”.

En el cuestionario se incluyeron ítems relacionados con las causas del absentismo. Los datos del análisis estadístico destacan que las faltas a clase se producían por dejarse llevar por otros compañeros, por las 
expulsiones que provocadas por sus problemas de conducta o porque tenían que ayudar en casa. Es de destacar que algunas chicas faltaban al colegio o al IES por esta última causa. Se puede estimar que hay familias que no dan importancia a la formación de las chicas; en especial aquellas en las que la madre carece de estudios. Según la investigación llevada a cabo por Sánchez et al. En 2008, el absentismo en los IES se producía por el aburrimiento hacia los contenidos escolares, un 29,0\%; por problemas de salud, un $16,0 \%$; por sanciones disciplinarias, un 11,8\%; porque los amigos tampoco iban, un 10,7\%; lo que corrobora en parte nuestro estudio, en el que se constata el bajo grado de motivación.

Los resultados obtenidos en esta investigación realizan un diagnóstico bastante claro de los puntos débiles en relación a las variables internas del sujeto y su relación con el fracaso escolar en la Ciudad Autónoma de Melilla.

Las nuevas líneas de investigación que se proponen van encaminadas a dar una respuesta educativa eficaz ante el diagnóstico realizado. Esta se podría concretar en:

- $\quad$ Programas educativos para las escuelas de familia.

- Cursos del profesorado con formación en factores afectivoemocionales que afectan al aprendizaje y formas de llevarlo a la práctica diaria del aula: motivación, expectativas, atribuciones, autoestima, estrategias para mejorar el clima social del aula, etc.

- C Cursos específicos sobre metodología en el área de matemáticas.

- $\quad$ Fomentar programas de educación emocional.

- $\quad$ Potenciar el control del estrés y la ansiedad a través de la enseñanza aprendizaje de técnicas de relajación en la materia de Educación física y en las actividades tutoriales.

\section{References:}

Antelm Lanzat, A.M.; Gil López, A.J.\& Cacheiro González, M.L., (2015). Análisis del fracaso escolar desde la perspectiva del alumnado y su relación con el estilo de aprendizaje. Educación y Educadores. 18, (3), p.471-489.

DOI: 10.5294/edu.2015.18.3.6

Alvarez Blanco, L. (2006). Familia y abandono escolar: Importancia de la implicación familiar en el proceso educativo. Madrid: Cinca.

American Psychiatric Association (2014). Guía de consulta de los criterios diagnósticos del DSM-5. Madrid: Editorial Médica Panamericana

García Gracia, M. (2013). Absentismo y abandono escolar: La persistencia de una problemática escolar y social. Madrid: Síntesis.

Barca Lozano, A. (2009). Motivación y aprendizaje en contextos educativos. Granada: Grupo Editorial Universitario. 
Benedet, M. J. (2014). Las dificultades de aprendizaje y-o adaptación escolar: Errores de diagnóstico y tratamiento: Sus graves consecuencias para el niño. Madrid: Ciencias de la Educación Preescolar y Especial.

Casquero Tomás, A., San Juan Solís, J. \& Antúnez Torres, A. School dropout by gender in the European Union: Evidence from Spain. Estudios Sobre Educación, (23), p.117-139.

Casquero Tomás, A. \& Navarro Gómez, M.L. (2010). Determinantes del abandono escolar temprano en España: un análisis por género. Revista de Educación, (2010), p. 191-223.

Castejón Costa, J. L., Navas Martínez, L., \& Ebrary, I. (2013). Dificultades y trastornos del aprendizaje y del desarrollo en infantil y primaria. Alicante: Ecu.

Carrillo Picazo, ML.M. (2009). La familia, la autoestima y el fracaso escolar del adolescente. Tesis doctoral inédita. Universidad de Granada. España.

Consejo Escolar del Estado. Ministerio de Educación, Cultura y Deporte. Informe sobre el estado del sistema educativo. Cursos 2013-2014. (2016). Extraído de http//ntic.educacion.es/cee/i2015cee.

Cordero Ferrera, J.M., Pedraja Chaparro, F., \& Simancas Rodríguez, R. (2015) Factores del éxito escolar en condiciones socioeconómicas desfavorables. Revista educación, (370), p. 172-187. doi: 10.4438/1988592X-RE-2015-370-302

Fernández Castillo, A. \& Gutiérrez Rojos, M.E. (2009). Atención selectiva, ansiedad, sintomatología depresiva y rendimiento académico. Electronic Journal of Research in Educational Psychology. 7, (17), p. 49-76.

Ferguson, E.D. (2000). Motivation: a biosocial and cognitive intergation of motivation and emotion. Great Britain: Oxford University Press.

Gamoran, A. (2001). American Schooling and Educational Inequality: A Forecast for the 21st Century. Sociology of Education, 74, p. 135-153

Garalgordobil, M. \& Duré, A. (2016). Relaciones del autoconcepto y la autoestima con la sociabilidad, estabilidad emocional y responsabilidad en adolescentes de 14 a 17 años. Análisis y Modificación de Conducta. 32 (141), p. 39.

García López, J. (2006). Aportaciones de la teoría de las atribuciones causales a la comprensión de la motivación para el rendimiento escolar. Ensayos (21), p. 217-232

Gutiérrez Tapias, M. (2003). La acción tutorial como alternativa para el desarrollo de la inteligencia emocional de los alumnos. San Cristóbal: Universidad de Los Andes. ULA.

Instituto Nacional de Evaluación Educativa. (2015). Panoramas de la Educación. Indicadores de la OCDE 2014. Ministerio de Educación, Cultura y Deporte. 
Marchesi, A. (2003). El fracaso escolar en España. Madrid: Fundación Alternativas

Martos Ortega, J. M., Domingo Segovia, J., Bolívar Botía, A. (2012). Estudiantes en riesgo de exclusión educativa en la enseñanza secundaria obligatoria de Andalucía. Granada: Universidad de Granada.

Ministerio de Educación, Cultura y Deporte (2015). Datos y Cifras del curso 2015-2016. Madrid: MECD

Organización para la Cooperación y el Desarrollo Económico, \& OECD. (2012). Panorama de la educación 2011: Indicadores de la OCDE. Madrid: Santillana, Spain.

O’Moor, M. \& Kirkham, C. (2001). Self steem and its relationship with bullying behavior. Aggresive behaviour. 27 (4), 269-283.

Ortega Ruiz, P., Mínguez Vallejos, R. \& Rodes Bravo, M.L. (2000). Autoestima: Un nuevo concepto y su medida. BIBLID 12, p. 45-46.

Ortega Torres (2006). Bajo rendimiento escolar. Bases emocionales de su origen y vías afectivas para su tratamiento. Madrid: Incipt editores

Pascual, L. C. \& Ebrary, I. (2010). Educación, familia y escuela. Rosario: Homo Sapiens.

Pintrich, P.R. (2003). A Motivational Science Perspective on the Role of Student Motivation in Learning and Teaching Contexts. Journal of Educational Psychology. (95), p.667-686.

Pintrich, P.R. \& Schunk, D.H. (2006). Motivación en contextos educativos. Teoría, investigación y aplicaciones. Madrid: Pearson Education.

PISA (2012). Programa para la Evaluación Internacional de Alumnos. Recuperado de www.mecd.gob.es/inee/estudios/pisa.html

Pulido Acosta, F., \& Herrera Clavero, F. (2015). Miedo, inteligencia emocional y rendimiento académico en el contexto educativo pluricultural de Ceuta. The UB Journal of Psychology. 45, (2), p. 249-263.

Rodríguez Fuentes, G. (2009) Motivación, estrategias de aprendizaje y rendimiento académico en estudiantes de E.S.O. Tesis doctoral inédita. Facultad de Ciencias de la Educación. Universidad de A Coruña. España.

Rosenthal, R. (1980). Pygmalión en la escuela: expectativas del maestro y desarrollo intelectual del alumno. Madrid: Marova

Ruiz de Miguel, C. (2001). Factores familiares vinculados al bajo rendimiento. Revista Complutense de Educación, 12, (1), p. 81-113.

Sánchez Fernández, S. et al. (2008). ¿Por qué dejé de estudiar?. El abandono escolar temprano en Ceuta y Melilla. Granada: Universidad de Granada

Sistema Estatal de Indicadores de la Educación. (2015). Madrid: Ministerio de Educación, Cultura y Deporte. 
Secretaría General Técnica: Ministerio de Educación. Instituto de Evaluación (2008). Informe 2008: Objetivos educativos y puntos de referencia 2010. Madrid: MECD.

VV.AA. (2005). El Protagonismo del profesorado. Experiencias de Aula y Propuesta para su Formación. Madrid: MECD.

VV.AA. (2008). El abandono escolar temprano en las ciudades de Ceuta y Melilla. Madrid: MECD.

VV.AA. (2010). Informe 2010 sobre el estado del sistema educativo. Curso 2008-2009. Ministerio de Educación. Consejo Escolar del Estado.

VV.AA. (2012). Informe 2012 sobre el estado del sistema educativo. Curso 2010-2011. Consejo Escolar del Estado. Madrid: MECD.

Weiner, B. (1986). An atributional theory of motivation and emotion. New York: SpringerVerlag

Weiner, B. (2000). Intrapersonal and interpersonal Theories of motivation from an atributional perspective. Educational Psycology Review (12), 1-14 